\title{
Source VUV à tube à éclairs
}

\section{Y. Vitel}

Laboratoire des Plasmas Denses, Université P. \& M. Curie, Tour 12 E5, 4 Place Jussieu, 75252 Paris Cedex 05, France

Abstracti We pregent a flagh tube light source providing a high intengity gpectrum in the vov range (170-250 $\mathrm{nm})$. The flash tube is filled with argon, krypton or xenon gas under an initial pxessure of 50 or 100 torrs. The vov spectrum is mainly due to intense ionic lines of the noble gas superposed on a weaker recombination continuum.

Nous avons réalisé une source de lumière à tube à éclairs émettant un fort rayonnement dans l'ultraviolet lointain (bande spectrale 170$250 \mathrm{~nm})$. Six tubes à enveloppe de silice synthétique à haute pureté, assurant une bonne transmission optique jusqu'a $170 \mathrm{~nm}$, de distance interélectrode de $12 \mathrm{~cm}$ 'et de $8 \mathrm{~mm}$ de diamètre intérieur ont été étudiés. Ils sont emplis respectivement de xénon, de krypton et d'argon sous des pressions initiales de 50 ou 100 torrs. Le circuit d'alimentation électrique de cette lampe permet d'obtenir des décharges d'une durée de l'ordre de $30 \mu \mathrm{s}$ à mi-hauteur avec des intensités de courant maximales variant de 500 à 2500 A et des fréquences de répétition de 1 à $50 \mathrm{~Hz}$.

La flgure 1 décrit le dispositif expérimental utilisé pour l'enregistrement des spectres UV: la lampe est placée dans une enceinte couplée hermétiquement à un monochromateur à la sortie duquel se trouve un photomultiplicateur. L'ensemble est pompé sous un vide primaire (1.10-2 torr) puis empli sous 1,1 bar d'azote pur. Un dispositif optique permet de faire l'image du tube sur la fente d'entrée du monochromateur. L'étalonnage en luminance du rayonnement est assuré par comparaison avec une lampe au deutérium calibrée par le laboratoire National de Métrologie du CNAM et placée dans les mêmes conditions géométriques que le tube à éclairs.

\section{$E_{1}$ Enceinte en inox \\ $\mathrm{E}_{2}$ Enceinte en laiton \\ D Diaphragme \\ $\mathrm{F}_{1} \mathrm{~F}_{2}$ Fentes d'entrée et de sortie \\ $\mathrm{R}$ Réseau concave VUV \\ L Lentille $\mathrm{MgF}_{2}$ \\ $T$ Tube à éclairs}

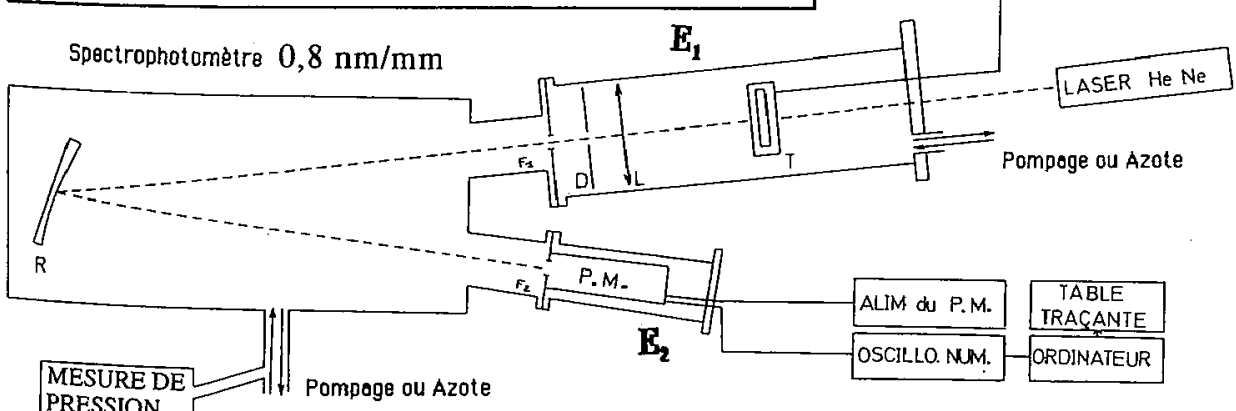

Figure 1: schéma đu đispositif expérimental 
Nous avons mesuré l'évolution du spectre d'émission dans l'ultraviolet lointain suivant différentes conditions de décharges. Celui-ci est principalement constitué de fortes raies spectrales de l'atome une fois ionisé du gaz considéré qui se superposent à un faible continuum comme le montre la figure 2 ci-dessous.

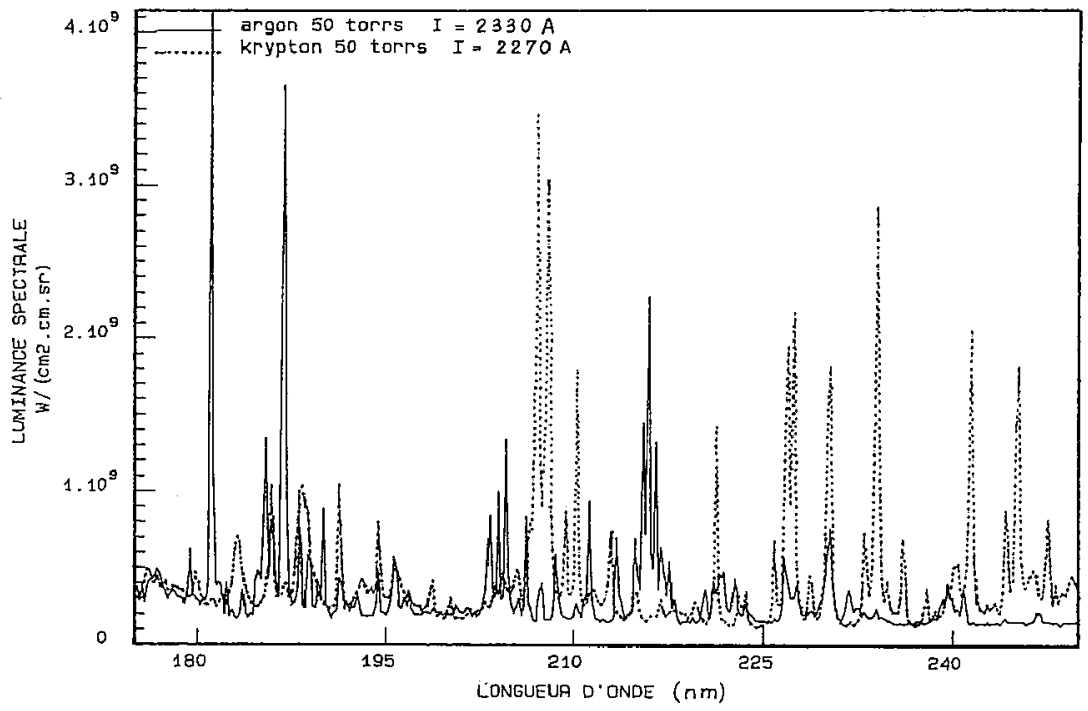

Figure 2: Exemple de spectres d'émission vuV

Le tableau I donne, comme exemple, les valeurs de la luminance maximale instantanée et de la luminance moyenne (à l Hertz de répétition des tirs), dans l'intervalle spectral de 175 à $200 \mathrm{~nm}$, pour les trois gaz à 50 torrs de pression initiale et différentes intensités de courant.

TABLEAU I

\begin{tabular}{|c|c|c|c|c|c|c|c|c|}
\hline \multicolumn{3}{|c|}{ Argon } & \multicolumn{3}{|c|}{ Krypton } & \multicolumn{3}{|c|}{ Xénon } \\
\hline$I(A)$ & $\begin{array}{l}L \max \\
\mathrm{w} /\left(\mathrm{cm}^{2} \mathrm{\theta x}\right)\end{array}$ & $\begin{array}{l}\mathrm{L} \text { moy } \\
\mathrm{m} \text { w/ }\left(\mathrm{cm}^{2} \mathrm{\theta r}\right)\end{array}$ & $\mathrm{I}(\mathrm{A})$ & $\begin{array}{l}\mathrm{L} \text { max } \\
\mathrm{w} /\left(\mathrm{cm}^{2} \mathrm{sr}\right)\end{array}$ & $\begin{array}{l}\text { L moy } \\
\mathrm{m} /\left(\mathrm{cm}^{2} \mathrm{Br}\right)\end{array}$ & $\mathrm{I}(\mathrm{A})$ & $\begin{array}{l}\text { L max } \\
\text { W/ }\left(\mathrm{cm}^{2} \mathrm{Br}\right)\end{array}$ & $\begin{array}{l}\mathrm{L} \text { moy } \\
\mathrm{m} \mathrm{w} /\left(\mathrm{cm}^{2} \mathrm{\theta r}\right)\end{array}$ \\
\hline 650 & 120 & 3,6 & 580 & 90 & 2,5 & 620 & 50 & 1,5 \\
\hline 980 & 180 & 5,0 & 900 & 150 & 3,9 & 870 & 110 & 3,2 \\
\hline 1280 & 310 & 8,7 & 1230 & 260 & 6,8 & 1170 & 250 & 7,0 \\
\hline 1630 & 490 & 13,2 & 1580 & 520 & 13,5 & 1460 & 330 & 9,2 \\
\hline 1980 & 770 & 20,8 & 1920 & 745 & 19,4 & 1750 & 550 & 16,0 \\
\hline 2330 & 780 & 20,3 & 2270 & 860 & 22,4 & 2100 & 790 & 23,0 \\
\hline
\end{tabular}

